\title{
F18-FDG Cardiac PET/CT: An Alternative Tool for Myocardial Viability Determination Prior to Coronary Revascularization Decision in Severe Ventricular Dysfunction
}

\author{
Süreyya Talay ${ }^{1}$, [MD] \\ ORCID: 0000-0002-4908-3850 \\ Nahide Belgit Talay ${ }^{2}$,[MD] \\ ORCID: 0000-0001-8483-2776
}

\section{nee) ABSTRACT Cen}

\begin{abstract}
Objective: The aim of our study was to evaluate clinical value and accuracy of Fludeoxyglucose Cardiac Positron Emission Tomography Computerized Tomography as an alternative tool for myocardial viability determination prior to coronary revascularization decision in lower left ventricular ejection fraction patients. Materials and Methods: Between the dates of 01.01.2010 and 10.07.2019, 191 consecutive patients (mean age $64 \pm 9.1$ years) underwent coronary artery bypass graft operations with severe left ventricular ejection fraction dysfunction with $35 \%$ or less. These impaired left ventricular ejection fraction patients were calculated as $4.4 \%$. Myocardial viability was studied by Fludeoxyglucose Cardiac Positron Emission Tomography Computerized Tomography for all cases. Final surgical decision was primarily depended on Fludeoxyglucose Cardiac Positron Emission Tomography Computerized Tomography for the majority of cases.

Results: 191 coronary artery bypass graft operations were performed. Perioperative deaths occured in 18 (9.4\%) cases. 236 patients with impaired left ventricular ejection fraction and coronary artery disease were evaluated by Fludeoxyglucose Cardiac Positron Emission Tomography Computerized Tomography prior to operation. 191 cases $(80.9 \%)$ were accepted as canditates for revascularization with multiple viable segments.45 cases (19.1\%) presented transmural scar tissue (non-viable) images by Fludeoxyglucose uptake analysis. This group cases were considered to be with non-beneficial results from revascularization. Thus, these patients were referred to medical treatments. Mean number of viable segments on Fludeoxyglucose Cardiac Positron Emission Tomography Computerized Tomography were calculated as $5.2 \pm 1.4$ for each patient.

Conclusions: The presence of myocardial viability is crucial to define reasonable canditates for revascularization in cases with lower left ventricular ejection fraction. Among other preoperative viability detection techniques such as echocardiography and myocardial perfusion scintigraphies, Fludeoxyglucose Cardiac Positron Emission Tomography Computerized Tomography is accepted as the 'Gold Standart' for segmental analysis on basis of distinguishing scar tissue from viable components. Key words: Miocardial viability, FDG-PET (CT), CABG, lower left ventrikular ejection fraction, preoperative surgery endication decision.

Key Words: Rib Fracture, Flail Chest, Rib Plate, Thoracic Traumas, Pain
\end{abstract}

Received:29 December 2019, Accepted: 03 March 2020,

Published online: 31 March 2020

\section{INTRODUCTION}

Coronary artery bypass graft (CABG) operations for patients with lower left ventricular ejection fraction (LVEF) is reported to be with improved longterm survival compared to medically treated patients [1]. However, these patients present higher mortality and morbidity percentages due to severe perioperative left ventricular dysfunction with low cardiac output and multiorgan dysfunction (MOD). For most of the cases, additive inotropic medication and mechanical support systems such as intraaortic counterpulsation balloon pumps (IABP) are required postoperatively in most cases. Prolonged cardiopulmonary bypass (CPB) lenght, postoperative arrhythmia, pulmonary dysfunction and renal insufficiencies, intubation periods, heart failure with MOD and intensive care unit treatments are not rare among this population. Nevertheless, surgical revascularization still remains the treatment of choice for 
coronary artery lesions with acceptable postoperative results.

Operation decision for lower LVEF cases depends on multiple variables. American Heart Association (AHA) criteria can be accepted as a guide for most cases. It is essential that the general condition of the patients by means of other organ system functions is a main subject on this manner.

On the other hand, myocardial viability plays a key role on postoperative response to revascularization. Therefore, in our daily practice, we study myocardial viability and segmental analysis of the ventricle prior to surgery to determine a decision for surgery in every case with severe cardiac dysfunction.

The aim of the present study was to evaluate clinical value and accuracy of Fludeoxyglucose Cardiac Positron Emission Tomography Computerized Tomography (F18-FDG PET/CT) as an alternative tool for myocardial viability determination prior to coronary revascularization decision in lower LVEF patients. Pathophysiological basis of this metabolic visualization depends on F18FDG-glucose combination utilization for myocardial energy supply. Myocardial energy substrate demand is responded via fatty acides in normal tissue perfusion conditions. Coronary artery disease existance and diminishing of blood supply result with enhanced levels of glucose uptake, intracellularly. Ischemic and/ or hibernating myocardial tissue glucose uptake is evaluated with F18 FDG marking. Thus, cardiac PET/ $\mathrm{CT}$ is with great importance for myocardial metabolism evaluation and viability determination.

We accepted lower LVEF as $35 \%$ or less for the study. Cardiac PET/CT is reported to be the gold standart for myocardial viability detection among other techniques such as thallium-201 rest-redistribution scintigraphy myocardial perfusion imaging (SPECT MIBI) and dobutamine stress echocardiography $[2,3,4]$.

\section{MATERIALS and METHODS}

Between the dates of 01.01.2010 and 10.07.2019, 191 consecutive patients (mean age $64 \pm 9.1$ years) underwent CABG operations with severe LVEF dysfunction with $35 \%$ or less.

Preoperative preparation included LVEF measurents by transthoracic echicardiography and/or catheter study for left ventriculography. All transthoracic echocardiographical evaluations presented multiple segments of hypokinetic and akinetic wall motion abnormalities. Mean value of LVEF in this patient population was $23.5 \% \pm 8.1$ (range $11 \%$ to $35 \%$ with a median value $29 \%$ ). Myocardial viability was also studied and elaborated by F18-FDG Cardiac PET/CT for all cases. Elaboration was detailed as viable myocardial tissue, hibernated myocardial tissue or necrosis areas (scars, nonviable tissue) via segmentally images of the heart. Final surgical decision was primarily depended on F18-FDG Cardiac PET/CT for the majority of cases. General indications were based on AHA acceptances such as left main coronary artery stenosis $\geq 50 \%$, left anterior descending artery(LAD) stenosis $\geq 70 \%$, three vessel stenosis, LAD stenosis $\geq 50 \%$ accompanied by a secondary coronary artery stenosis. Lower LVEF was not accepted as a contra-indication for surgery at any level unless a viable tissue was demonstrated by F18-FDG Cardiac PET/CT. Cases with reports of scar tissue (no evidence of myocardial tissue viability) from F18-FDG PET/CT scans were not operated. Because we believe that benefits may only be gained via demonstrated existence of viable myocardial tissue for these patients. Otherwise, revascularization may not be reasonable.

Depending on New York Heart Association (NYHA) criteria; 89(46.5\%) patients were functional class IV, $81(42.4 \%)$ patients were class III and 21(10.9\%) (the sum of percentages is 99.9) patients were class II. All patients had a previous myocardial infarction history ( $\mathrm{n}=191,100 \%)$. Besides, 104 (54.4\%) patients reported a cardiological percutaneous intervention with balloon angioplasty and/or coronary stenting. Refusing a surgical decision by patients' personal reasons during previous angiographies was one of the most frequent conditions among this group of cases.

Chronic obstructive pulmonary disease (COPD) with previous medication history was recorded in 35 patients (18.3\%). Diabetes mellitus was the most common co-morbidity with 58 cases (30.3\%). Preoperative hemodialysis (HD) necessities were observed in 29 (15.1\%) patients. As a clinical protocol, routine $\mathrm{HD}$ at the first postoperative day was 
planned for this group when haemodynamic conditions were acceptable, as a clinical protocol. Emergency CABG cases without F18-FDG Cardiac $\mathrm{PET} / \mathrm{CT}$ scanning were excluded from the study. These cases were preoperatively evaluated by ventriculography and/or transthoracic echocardiography, solely. Furthermore, thallium-201 rest-redistribution scintigraphy myocardial perfusion imaging and single photon emission computed tomography (SPECT MIBI) and dobutamine stress echocardiography without F18-FDG C-PET/CT cases were also not included to this present study.

Additionaly, cases with concomitant cardiac procedures such as valve surgery, aneurysmectomy, major vascular procedures and re-operations were excluded from the study. We aimed to refine the patient population in order to focus on F18 -FDG Cardiac PET/CT accuracy and useability on this specific group of patiens as an alternative to SPECT MIBI or dobutamine stres echocardiography.

Preoperative F-18-FDG Cardiac PET/CT images and coronary angiography correlation (bold)

PET is a nuclear medicine practice to evaluate functional processes by 3D images. The system is based on gamma rays that is emitted indirectly by a positron emitting radionuclide tracer. In a PET and CT combination, CT x-ray scanning is also performed at the same session. PET and CT scans intersect at the same section with more elaborate anatomical determination. For FDG PET/CT, biological active molecule is FDG which is a glucose analogue. Thus, FDG represents tissue metabolic activity by means of glucose uptake. Glucose uptake also is the most certain evidence of tissue viability. It is well described and reported that FDG PET/CT is accepted as the gold standart for exhibiting cardiac viability and/or hibernating myocardium and also for necrosis discrimination $(4,5)$. On the other hand, PET/CT is definitively non-invasive but it certainly does involve exposure to ionizing radiation, especially with $\mathrm{CT}$ combinations.

Prior to imaging, we continued preoperative medications of diuretics, angiotensin converting enzyme inhibitors, nitrates and calcium antagonists. We ceased aspirin and antianginal medications 3 days prior to imaging and operations.

For our primary goal of the study, myocardial glucose usage was evaluated by F18-FDG PET/CT during hyperinsulinemic euglycemic clamping. Images were taken in a glucose-loaded state after $50 \mathrm{~g}$ glucose administration. As a routine application, we continued with a 25 minute transmission scan for correction of photon attenuation. Transaxial images were obtained for 25 minutes for each case. We applied FDG doses in $10 \mathrm{mCi}$ intravenously. After 30 minutes of waiting period we scanned images for FDG uptake at least for 20 minutes. Image analyis and reconsctructions were performed with Apple Comp. Inc. Macintosh Afterwards FDG polar maps were compared with accepted normality modules by means of identifying hypoperfused and/or aperfused segments which are correlated with hybernating and/ or viable tissue to scar formation. Synchronous CT scan sections with FDG PET images were allowed us to express for each coronary vascular territory specifically. In order to achieve this evaluation, anatomical and regional perfusion data were stored in a 64X64, 16 bit matrix. By our raw PET data, $6 \mathrm{~mm}$ thick (1 pixel) transaxial slices were reconstructed corrected for attenuation with $\mathrm{CT}$ images.

A ROI based method was also administered for quantitative evaluation. Segmentally highest F18 FDG accumulation accepted as a reference line $(\geq 70 \%)$ for normal viable myocardial tissue. Thus, visual data for F18 FDG uptake was reported in 3 different classifications for segmental viability; (0): Scar tissue without viability (infarct, a ROI calculation value $<50 \%)$, (1):peri-infarct viability probably hibernating myocardial tissue (a ROI calculation value from $50 \%$ to $70 \%$ ), (2): normal viable myocardial tissue $(\geq 70 \%)$. For segmental analysis of F18-FDG Cardiac PET/CT, we evaluated anterior, anterolateral, lateral, inferolateral, inferior, inferoseptal, septum, anteroseptal, apex segments for each patient and each investigation.

Depending on the FDG uptake concordantly perfusion evaluation segments were described by experienced specialists of Nuclear Medicine. 
Echocardiographically dysfunctional segments with a FDG uptake reductions were considered nonviable and vice versa. Subendocardial and myocardial scars were defined as FDG uptake mismatches.

This study is made under official approval and permission of our hospital administrators. The Ethical committees of hospitals approved the study protocol (2009-CT7-038), and all procedures were carried out in accordance with the Declaration of Helsinki (supl.October / 2008).

\section{Data Collection}

Preoperative demographical data was recorded with perioperative variables such as age, sex, COPD, DM, co-morbidities, echocardiographical measurements including LVEF, coronary angiography results, graft number per case, cross clamp time (CCT), CPB lenght, intensive care unit (ICU) and total hospitalization lenght, (perioperative) deaths, perioperative $H D$ incidence and IABP insertions.

$2.0 \mathrm{~L} /$ minute/m2 cardiac index was accepted as a threshold for low cardiac output syndrome and indication for IABP insertion. Inotropic medication consisted of dopamine, dobutamine, norepinephrine and epinephrine infusions with seldom administrations of levosimendan.

Decisions for postoperative HD indications were reached by co-operations from nephrologists for each case seperately.

F18-FDG Cardiac PET/CT scanning was performed with Siemens Corp. Inc, model 931-108 units. All images were studied by experienced specialist for nuclear medicine and results were consulted by surgeons prior to surgical decision. Segmental analysis for viability was consisted of seperate investigations on evaluated anterior, anterolateral, lateral, inferolateral, inferior, inferoseptal, septum, anteroseptal, apex segments. Afterwards, we compared these nuclear scanning results with coronary angiographical occluded segments to understand the feasibility of revascularization. For instance, anterior wall akinetic findings from echocardiography with left anterior descending artery (LAD) occlusion on coronary angiography was specifically questioned by anterior segment viability via F18-FDG Cardiac PET/CT.
Operative mortality was defined as deaths within 30 days. Perioperative deaths accepted as deaths during postoperative hospitalization period. However, mortality records after discharge from the hospital may not reflect the exact survival. Because our patients come from a wide geographical area and some patients prefer to continue their treatment at their hometown hospitals. Nevertheless, it was possible for us to reach the majority of the population ( $n=183,95.8$ ) among study group during the survival follow-ups. On the other hand, neither the clinical outcome nor the intraoperative data was our primary aim for the study.

Study data essentially aimed to address the preoperative utility possibilities for F18-FDG Cardiac PET/CT for lower LVEF CABG indications, not to determine the correlation of survival to any other variable.

\section{Statistical analysis}

Differences in continuous variables were analyzed by Student $t$ tests. The data are expressed as proportions or as the mean \pm standard deviation (SD). Differences in categorical variables were analyzed by 2 analysis. Statistical modeling was performed using S-PLUS statistical software (MathSoft, Cambridge, MA ).

\section{RESULTS}

\section{Preoperative and Surgical procedures}

Preoperative premedication was applied with Propofol 2-3 mg / kg, Fentanyl Citrate 10-15 microgr / kg and Pancuronium bromid 0,1 mg / kg. Maintenance dose included Propofol 2-5 mg / kg / h and Fentanyl Citrate 5-10 microgr / kg / h IV. Inhaled anaesthesia continued by Sevoflurane. Lung ventilation performed via $0.4 \mathrm{Fi} 02$.

All patients underwent surgery that was mainly based on coronary evaluations by coronary angiography. Following median sternotomy, left intermammarian artery (LIMA) and great saphenous vein (VSM) grafts were prepared. Heparin administered with 350 units / per kilogram. Operative activeted clotted time (ACT) aimed at least 400 seconds. 
After pericardiectomy, aorta-caval cannulation performed. Within 28 centigrade degrees of body core temperature, cardiopulmonary bypass was started with centrifugal pump (Bio-Pump BPX-80, Medtronic, Medtronic. Inc, Minneapolis, USA) and membrane oxygenator (Sorin-Biomedica, Dideco, d 708 simplex II, Italy). Myocardial protection was used in three steps of cardioplegia: $500 \mathrm{ml}$ of normothermic blood cardioplegia, cold cardioplegia in $10 \mathrm{ml} /$ per $\mathrm{kg}$ in $40 \mathrm{mmHg}$ pressure and 500 $\mathrm{ml}$ of hot shot before removal of the cross clamp. Additive cardioplegia doses continued in every 20 minutes as a clinical protocol. Topical cooling was applied during initial phase cardioplegia. Coronary anastomoses were performed under cardiac arrest. Following the removal of cross clemp and protamine sulphate administration, proximal anastomoses were performed with a side-biting clamp

Table 1. Preoperative and demographic parameters to aorta. Other CABG procedures such as beating heart were not applied for any case. Ages over 70 years was considered as a threshold for definite LIMA graft indication.

Weaning period from CPB was achieved with additive support from epinephrine $(0.02$ to $0.1 \mathrm{micg} / \mathrm{kg} /$ $\mathrm{min}$ ) and norepinephrine (0.02 to $0.1 \mathrm{micg} / \mathrm{kg} / \mathrm{min}$ ) when indicated. CPB weaning diffuculties despite the administration of inotropic agents accepted as a general indication for IABP when possible. During the ICU follow-up, lower values than $2.0 \mathrm{~L} /$ minute/ $\mathrm{m} 2$ of cardiac index with clinical features of heart failure were also accepted as IABP indications.

\section{Patient characteristics}

Preoperative data and demographical presentations of our study group are summarized in Table 1.

\begin{tabular}{|l|c|}
\hline Variable & Value (Mean/SD) \\
Total CABG performed, $\mathrm{n}$ & 191 \\
Mean age (year) & $64.9 \pm 9.1$ \\
LVEF <20\%,n(\%) & $37(19.3 \%)$ \\
F18-FDG C-PET/CT, n(\%) & $191(100 \%)$ \\
NYHA Class III, n(\%) & $81(42.4 \%)$ \\
NYHA Class IV, n(\%) & $89(46.5 \%)$ \\
COPD, n(\%) & $35(18.3 \%)$ \\
DM, n(\%) & $58(30.3 \%)$ \\
HD, n(\%) & $29(15.1 \%)$ \\
Preoperative Inotrop Dependency, n(\%) & $27(14.1 \%)$ \\
Previous CABG & $21(10.9 \%)$ \\
\hline
\end{tabular}

Values presented are mean \pm SD. BMI: Body mass index, CABG: Coronary artery bypass graft, COPD: Chronic obstructive pulmonary disease, DM: Diabetes Mellitus, F18-FDG C-PET/CT: Fluorodeoxyglucose 18 cardiac positron emission tomograpghy computerized tomography, HD: Hemodialysis, LVEF: Left ventricular ejection fraction, NYHA: New York Heart Association, MI: Myocardial infarction

All operations ( $n=191$ ) were performed with CPB and aortic cross clamp. Our routine protocol was used by all surgeons at the same fashion. Proximal anastomoses were finished by a side biting aort clamp to reduce the cross clamp lenghts. Swan-Ganz catheters were inserted as a routine for all cases to eavluate cardiac index as a component of patient follow-up.

Table 2 depicts our perioperative data from the study including intraoperative parameters and early postoperative follow-up details. 


\begin{tabular}{|l|c|}
\hline Variable & Value (Mean/SD) \\
Operation lenght (minute) & $281.2 \pm 33.5$ \\
Coronary graft number & $3.5 \pm 0.8$ \\
CPB time (minute) & $152.5 \pm 41.3$ \\
ACC time (minute) & $88.3 \pm 11.4$ \\
ICU stay (day) & $6.2 \pm 3.1$ \\
Overall mortality $\mathrm{n}, \%$ & $18(9.4 \%)$ \\
\hline
\end{tabular}

Values presented are mean \pm SD. ACC: Aortic cross clamp time, CPB: Cardiopulmonary bypass time, ICU: Intensive care unit

Perioperative deaths occured in $18(9.4 \%)$ cases. Mortality reasons were prolonged CPB in 4 (2\%) cases, severe ventricular arrhytmias in $5(2.6 \%)$ cases, MOD with prolonged intubation in 6 (3.1\%) cases and major neurological complication in $2(1 \%)$ cases. Our mean coronary graft number was $3.5 \pm 0.8$. LIMA was used in the majority of cases ( $n=176,92.1 \%)$. LIMA was anastomosed to LAD for each case.

IABP insertions were applied in $81(42.4 \%, 43$ cases intraoperatively, 38 cases at ICU) patients.

Afterwards, as a routine preoperative evaluation, segmental FDG uptake anaylysis was compared to coronary angiography results to reach a surgical decision (shown in Figure 1).

FDG cardiac PET/CTof Figure 1 was reported as anteroapical, anteroseptal, apex, mid and apical segments with normal FDG uptake and therefor normal metabolism. Inferior wall was observed with normal metabolic activity by means of hibernating myocardium. This patient was operated due to PET/CT results with viable myocardial tissue. Postoperative period was uneventful and the patient was discharged at the postoperative day 21 .

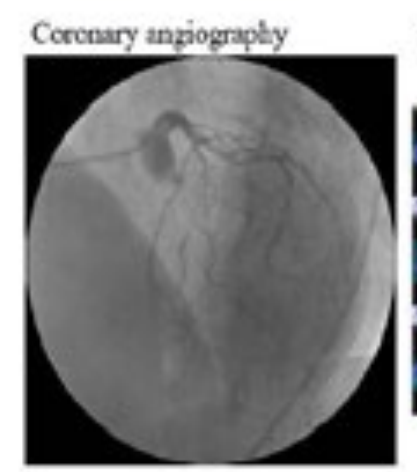

FDG andias PET-CT
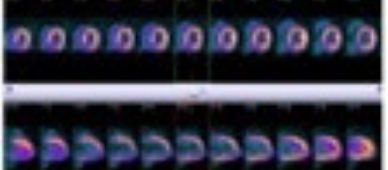

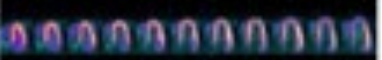

Figure 1. Case sample 1 with coronary angiography and preoperative F18 FDG c-PET/CT
Approved existence of scar tissue without any FDG uptake by segmental analysis from cardiac PET/CT report was accepted as a relative contraindication for revascularization (shown in Figure 2.).

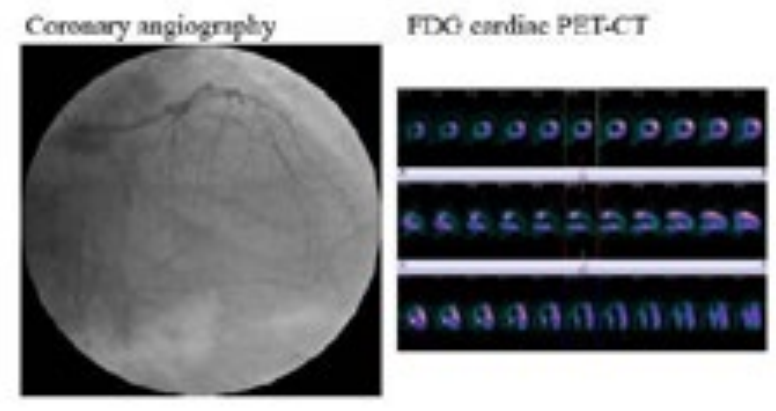

Figure 2. Case sample 2 with coronary angiography and preoperative F18 FDG c-PET/CT

FDG cardiac PET/CTof Figure 2 was reported as anteroapical, anteroseptal, inferoapical, apex, inferior wall mid and apical segments ametabolism (scar tissue). Viable and/or hibernating myocardial tissue was not observed. This case was not operated. Because we believed that the lack of preoperative viable myocardial tissue makes the revascularization non-beneficial.

236 patients with impaired LVEF and severe coronary artery disease were evaluated by F18-FDG Cardiac PET/CT prior to operation. 191 cases (80.9\%) were accepted as canditates for revascularization with multiple viable segments.

45 cases (19.1\%) presented transmural scar tissue (non-viable) images by FDG uptake analysis. This group cases were considered to be with 
non-beneficial results from revascularization. Thus, these patients were referred to medical treatments. Mean number of viable segments on F18-FDG Cardiac PET/CT were calculated as $5.2 \pm 1.4$ for each patient. Segmental viability dispersions were depicted in Figure 3.

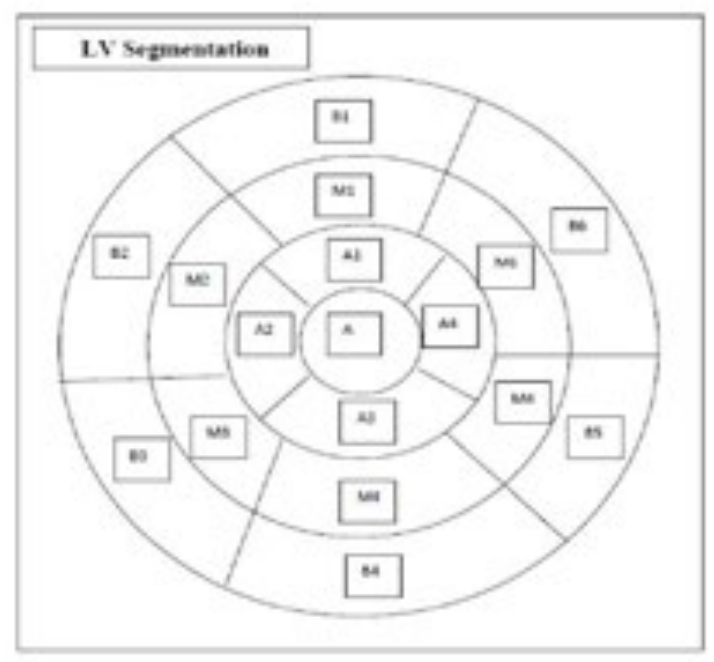

Figure 3. LV segmentation for FDG PET images

Left ventricle viability evaluation at Figure 3 presents polar-plot depiction according to 17 segments analysis; B1 (basal anterior), B2 (basal anteroseptal), B3 (basal inferoseptal), B4 (basal inferior), B5 (basal inferolateral) , B6 (basal anterolateral), M1 (mid anterior), M2 (mid anteroseptal), M3 (mid inferoseptal), M4 (mid inferior), M5 (mid inferolateral), M6 (mid atreolateral), A1 (apical anterior), A2 (apical septal), A3 (apical inferior), A4 (apical lateral) and A (apex) segments.

Equivalent coronary artery territories on short axis projections to polar-plot depictions to segmental analysis are presented in Figure 4.

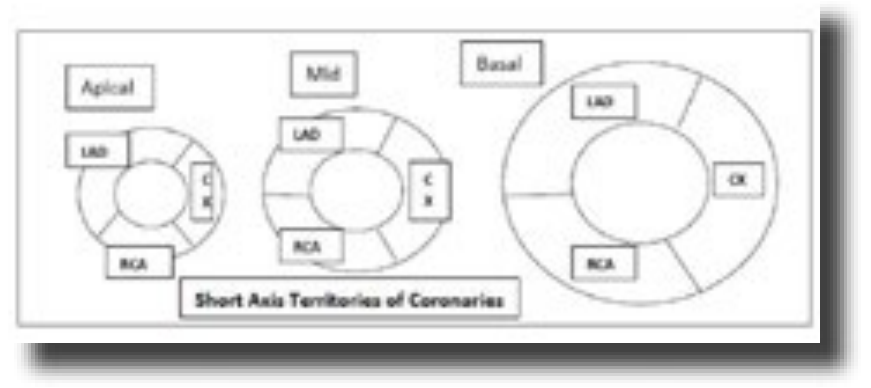

Figure 4. Coronary artery territories on short axis sections

Figure 4 presents three individual short axis slices corresponding to relevant coronary artery territory. As a combination of Figure 3 and Figure 4, we detailed viable segment percantages with meaningful FDG uptake on PET/CT from the study group in Table 3.

Table 3. Percentages of viable tissue evidence on segmental analysis at short axis images

\begin{tabular}{|c|c|}
\hline Segment & Quantity/percentage \\
\hline Basal anterior n, \% & $103 / 53.9 \%$ \\
\hline Basal anteroseptal n, \% & $106 / 55.4 \%$ \\
\hline Basal inferoseptal n,\% & $91 / 47.6 \%$ \\
\hline Basal inferior n,\% & $93 / 48.6 \%$ \\
\hline Basal inferolateral n,\% & $84 / 43.9 \%$ \\
\hline Basal anterolateral n,\% & $106 / 55.4 \%$ \\
\hline Mid anterior $n, \%$ & $181 / 94.7 \%$ \\
\hline Mid anteroseptal n,\% & $159 / 83.2 \%$ \\
\hline Mid inferoseptal n,\% & $148 / 77.4 \%$ \\
\hline Mid inferior $\mathrm{n}, \%$ & $62 / 32.4 \%$ \\
\hline Mid inferolateral n;\% & $75 / 39.2 \%$ \\
\hline Mid anterolateral n;\% & $92 / 48.1 \%$ \\
\hline Apical anterior $n, \%$ & $183 / 95.8 \%$ \\
\hline Apical septal n,\% & $179 / 93.7 \%$ \\
\hline Apical inferior $n, \%$ & $113 / 59.1 \%$ \\
\hline Apical lateral n,\% & $103 / 53.9 \%$ \\
\hline Apex n,\% & $125 / 65.4 \%$ \\
\hline
\end{tabular}


Values presented are quantity and percentage (assessmenst over patients with surgery, patients with medical treatment decisions were excluded from this extract )

LAD territories from the short axis apical, mid and basal sections (mainly B1, B2, M1, M2, A1, A2 areas from Figure 3.) viability assessments were accepted as necessary indications for CABG, parallel to AHA indications for coronary angiographies. Non-viable scar tissue without FDG uptake on LAD segments at FDG PET/CT images points out a previous severe myocard infarction on LAD feeding territory with a wide necrosis. A revascularization surgery in the latter case accepted to be non-beneficial.

\section{DISCUSSION}

Severe LVEF detorioration caused by severe coronary artery disease is with a poor prognosis in 5 years clinical follow-up. However, surgical treatment is also reported to be with higher mortality and morbidity rates $[5,6,7]$. Besides CABG, cardiac transplantation arises as an alternative solution with more acceptable clinical results for selected patients. But in general practice of cardiac surgery for these patients, revascularization with CABG still remains the best strategy with greatest benefit for this group. It is a proven fact that coronary revascularization improves the life quality on exercise tolerance and survival for patient with lower LVEF in the longterm trail compared with medical treatment. Remaining viable myocardium play a key role for revascularization decisions. F18-FDG Cardiac PET/CT, thallium-201 scintigraphy and dobutamine echocardiography are noninvasive prediction scanning techniques to put forth the presence of hibernating myocardium, viability and possibilities to recover contractile function after tissue revascularization. Evidences of viability may enlighten the phenomenon of myocardial hibernation that may raise the possibility of regaining functional recovery, even in LVEF. On the other hand, absence of approvable myocardial viability, it is most likely for a patient not to improve better LVEF after CABG. For the latter group of cases, postoperative clinical status and functional capacity is unclear [8].
Several studies aimed to distinguish between dysfunctional but also viable myocardial tissue and dysfunctional scar tissue in order to manage decisions in patients with ischemic cardiac episodes resulting with lower LVEF. Thallium 201 scintigraphy and SPECT can be applied in such a target patient population with higher accuracy and sensitivity. Dobutamine echocardiography can also produce clinically meaningful preoperative improvements on viability determination with a similar rates of accuracy with thallium scannings. SPECT analysis was reported to be with around $85-90 \%$ sensitivity and $80-95 \%$ specifity in several reports [9].

In this study, we used F18-FDG C-PET/CT to determine preoperative myocardium viability for its higher sensitivity and specifity.

Bax et al reported that viable segments on nuclear medicine techniques was not the only predictor of improvement in LVEF after revascularization [10]. Nonviable segments and Q-wave myocardial infarctions are other main factors contributing with postoperative out-come. Nevertheless, he reports two or more viable segments on FDG PET may present better revascularization result. He also signed that clinical healing by improving quality of life was also correlated with postoperative LVEF improvements. $5 \%$ or more LVEF improvement was considered to be with clinical better observations. Moreover, Bax pointed out that the presence of substantial viability was highly predictive of improvement in heart failure symptoms after revascularization. Di Carli and his co-workers reported a similar result by their study that the magnitude of improvement in heart failure symptoms after revascularization was related to the preoperative extent of viability assessed by FDG PET [11] . He pointed out that preoperative quantification of myocardial viability may be clinically useful to identify those patients who will benefit most from revascularization both functionally and prognostically. Di Carli et al assessed the relation between the magnitude of improvement in heart failure symptoms after CABG and the extent 
of myocardial viability assessed by use of quantitative analysis of preoperative PET images. PET results and postoperative healing comparison rates were reported by Di Carli for $76 \%$ in sensitivity and $78 \%$ in specifity on flow-metabolism PET mismatch for predicting improvement in symptoms of heart failure. In our study we prefered not to operate patients with preoperative PET/CT evidences of global scar tissue without viable segments. As a conclusion, he stated that preoperative myocardial vaibility is predictive of the degree of improvement clinically. Parallel to our study, he advocated PET to be with atmost beneficial gain in preoperative evaluation with higher accuracy rates.

In our experience, similar to the study of Domanski, existence of diabetes is a strong predictor of higher incidence of perioperative mortality and morbidity for patients with LV dysfunction [12]. Diabetes mellitus was the most common co-morbidity with 58 cases (30.3\%) from our study data. But our mortality rate was recorded in $18(9.4 \%)$ cases. 11 cases were premedicated for diabetes which represents a rate for DM in mortality group with a percantage of $61.1 \%$. Additionaly, 6 of these patients were redo CABG (33.3\%) cases. From our point of, parallel to recent medical reports, previous history of CABG is correlated with higher mortality risk as an independent factor $[13,14]$.

On the other hand, there are several reported unlear and contraversial points on this topic, such as STICH study from Velazquez et al [15]. He advocates that given the overall benefit noted with CABG in these patients over medical management alone, this argue against routine viability testing in all patients with ischemic cardiomyopathy prior to considerations for revascularization. He depicts that It is unclear though if the lack of benefit with myocardial viability testing note in this analyse represents a lack of power and a lack of sensitivity of testing tools utilized. Despite the study of Velazquez and his statement, it is clear that an F18-FDG Cardiac PET/CT is mentioned as 'Gold Standart ' of myocardial viability in several studies $[16,17]$. The latter data is mandatory for a CABG indication decision while a scar tissue does not require revascularization.

We believe that preoperatively identified glucose active myocardial tissue on F18-FDG Cardiac PET/ $\mathrm{CT}$ indicates the presence of viable tissue. Thus, the aim of the study is to accept these viable segments for indications of surgery. We suggest that F18-FDG Cardiac PET/CT can be applied as an alternative precious preoperative tool in cases with lower preoperative LVEF prior to CABG. LAD watering territories from the short axis apical, mid and basal sections should be primary target areas to investigate to determine feasibility of a LIMA-LAD anastomoses, which is one of the essential goals of CABG. Additive viable segments for right coronary artery and circumflex artery on F18-FDG C- PET/CT strenghten revascularization indication. On the contrary, proven nonviable scar tissue for LAD territory on F18-FDG Cardiac PET/CT may suspend surgical decision in most of the cases with reduced LV function prior to CABG whether with existance of viability evidences at right coronary artery and circumflex artery territories or not.

\section{LIMITATIONS of STUDY}

In this present study, we elaborated the preoperative importance of F18-FDG Cardiac PET/CT with perioperative parameters. However, our study does not present the long-term follows-ups of our operated patients. This point may require further investigations and multidisciplinary co-operations for instance by 6 months and yearly survivals of this patient group, post-CABG period. Thus, we could be able to prove survival value of F18-FDG Cardiac PET/ CT.

\section{CONCLUSION}

The presence of myocardial viability is crucial to define reasonable canditates for revascularization in cases with lower LVEF. Among other preoperative viability detection techniques such as dobutamin echocardiography and myocardial perfusion scintigraphies, F18 FDG cardiac PET/CT is accepted as 
the 'Gold Standart' for segmental analysis on basis of distinguishing scar tissue from viable components. We strongly advocate F18-FDG cardiac PET/ $C T$ to be a suitable and effective tool to evaluate CABG indications in severe ventricular dysfunction.

\section{CONFLICT OF INTEREST}

The authors declare that they have no conflict of interest.

\section{FUNDING}

This work was not supported financially by any third parties.

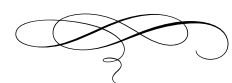


[1] Mickleborough LL, Maruyama H, Takagi Y, et al. Results of revascularization in patients with severe left ventricular dysfunction. Circulation 1995;92:73-79

[2] Pagano D, Bonser RS, Townend JN, et al. Predictive value of dobutamine echocardiography and positron emission tomography in identifying hibernating myocardium in patients with postischaemic heart failure. Heart 1998; 79: 281-88

[3] Pagano D, Townend JN, Littler WA et al. Coronary artery bypass surgery as treatment for ischemic heart failure: the predictive value of viability assessment with quantitative positron emission tomography for symptomatic and functional outcome. J Thorac Cardiovasc Surg 1998;115:791-99

[4] Strauss HW, Miller DD, Wittry MD, et al. Procedure Guideline for Myocardial Perfusion Imaging. J Nucl Med 2008; 36(3):155-61.

[5] Klocke FJ, Baird MG, Lorell BH. ACC/AHA/ASNC guidelines for the clinical use of cardiac radionuclide imaging: executive summary-a report of the American College of Cardiology/American Heart Association task force on practice guidelines (ACC/AHA/ASNC committee to revise the 1995 guidelines for the clinical use of cardiac radionuclide imaging). Circulation 2003;108:1404-18.

[6] Kennedy JW, Kaiser GC, Fisher LD, et al. Clinical and angiographic predictors of operative mortality from the Collaborative Study in Coronary Artery Surgery (CASS). Circulation 1981;.63:793-02.

[7] Passamani E, Davis KB, Gillespie MJ, et al. A randomized trial of coronary artery bypass surgery. Survival of patients with a low ejection fraction. N Engl J Med 1985;312:1665-71.

[8] Toda K, Mackenzie K, Mehra MR, et al. Revascularization in severe ventricular dysfunction (15\% LVEF 30\%): a comparison of bypass grafting and percutaneous intervention. Ann Thorac Surg 2002;74: 2082-87.
[9] Koepfli P, Hany TF, Wyss CA. CT attenuation correction for myocardial perfusion quantification using a PET/CT hybrid scanner. J Nucl Med 2004;45: 537-42.

[10] Bax JJ, Visser FC, Poldermans D. Relationship between preoperative viability and postoperative improvement in LVEF and heart failure symptoms. J Nucl Med 2001;42:79-86

[11] Di Carli MF, Asgarzadie F, Schelbert HR. Quantitative relation between myocardial viability and improvement in heart failure symptoms after revascularization in patients with ischemic cardiomyopathy. Circulation 1995;92: 3436-44.

[12] Domanski M, Krause-Steinrauf $H$. The effect of diabetes on outcomes of patient with advenced heart failure in BEST trial. J Am Coll Cardiol 2003; 42:914922.

[13] Topkara VK, Cheema FH, Kesaramanujam S. Coronary artery bypass grafting in patients with low ejection fraction. Circulation 2005;112:344-50.

[14] Appoo J, Norris C, Merali S. Longterm outcomes of isolated coronary artery bypass surgery in patients with severe left ventricular dysfunction. Circulation 2004;110:13-17.

[15] Robert HJ, Harvey W, Eric JV, Linda KS, et al. Surgical Treatment for Ischemic Heart Failure (STICH) Trial. Enrollment J Am Coll Cardiol 2010; 56(6): 490-98.

[16] Santos BS, Ferreira MJ. Positron emission tomography in ischemic heart disease. Rev Port Cardiol 2019; 38(8): 599-08.

[17] Fukushima $K$, Arashi $H$, Minami $Y$ et al. Functional and metabolic improvement after coronary intervention for non-viable myocardium detected by $18 \mathrm{~F}$ fluorodeoxyglucose positron emission tomography. J Cardiol Cases 2019;20(2): 57-60. 